\title{
Question on Some Principles of Electromagnetism
}

\author{
Kexin $\mathrm{Yao}^{1}$ \\ ${ }^{1}$ Institute of Mechanical Engineering of Shanxi Province, Xi'an, China \\ Correspondence: Kexin Yao, Institute of Mechanical Engineering of Shaanxi Province, Room 1-7-1, Staff \\ Building, Xi'an Metering Instiution, No. 12, Laodong South Road, Xi'an 710068, China. Tel: 86-134-8462-6424. \\ E-mail: yayydwpq@163.com
}

Received: April 15, 2012 Accepted: May 9, 2012 Online Published: July 20, 2012

doi:10.5539/apr.v4n3p115 URL: http://dx.doi.org/10.5539/apr.v4n3p115

\begin{abstract}
In theory of electromagnetism, there are principles indicated as below: motional magnetic field shall not exist; action and reaction can be in the same direction, but differ in value; magnetic field can have an infinitely great motional speed; the same physical phenomenon can be explained conversely in different inertia systems; there is no uniform scientific explanation for deflection of high-speed charged particle in magnetic field; coexistence of static magnetic field with static electric field cannot be explained. Hence, truth of the above principles of electromagnetism is questioned here.
\end{abstract}

Keywords: magnetic field, Lorentz force, special theory of relativity, induced electric field, self-induced electric field

\section{Motional Magnetic Field shall not Exist}

It is well known that the stator of three-phase asynchronous motor after being connected to three-phase alternating current will generate a rotating magnetic field which produces the completely same rotating magnetic effect as magnetic " $\mathrm{N}$ " and "S" stators do in rotating. According to Biot-Savart law, however, all current-carrying coils of stator of three-phase asynchronous motor can only produce a static magnetic field with magnetic induction constantly varying instead of a motional magnetic field. The above presents the situation when magnetic source (motor stator) remains static, while magnetic source motions, there is still the deduction that magnetic should be static according to Biot-Savart law.

In Figure 1, rectangle current-carrying coil abcd motions at speed $V$, it is naturally considered that the magnetic field produced by abcd should also motion at speed V; however, the analysis of electromagnetic fundamental principles shows that the magnetic field produced by coil abcd should be static only with the intensity of magnetic field varying.

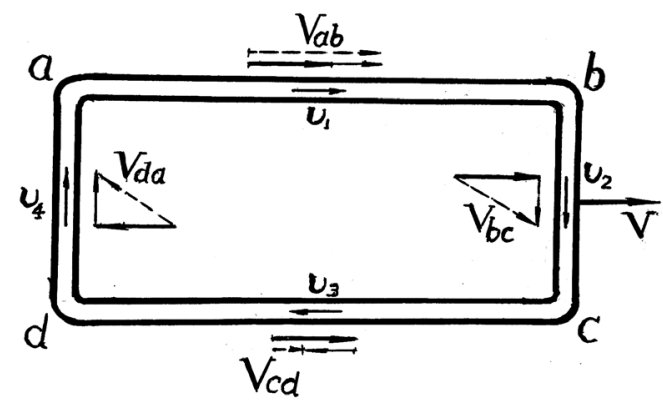

Figure 1. The motional current-carrying coil should produce a static varying magnetic field

In Figure 1, the average speed of free electron in coil relative to conducting wire is expressed as $v_{n}$, the speed of free electron along section ab relative to our complex motional speed should be $\mathbf{V}_{\mathrm{ab}}$; in the similar way, the complex motional speed of free electron along sections bc, cd and da shall be $\mathbf{V}_{\mathrm{bc}}, \mathbf{V}_{\mathrm{cd}}$, and $\mathbf{V}_{\mathrm{da}}$.

According to Biot-Savart law, the magnetic field generated by motional electrified body is static, thus the entire 
coil, namely the magnetic field generated by free electrons in leads ab, bc, cd and da certainly keep static at any time. In addition, the motional speed of entire coil and positively charge protons equivalent to electric quantity of free electron is $\mathrm{V}$, and its magnetic field certainly keeps static. It will be seen from this that the magnetic field generated by the entirely motional current-carrying coil should be static, but the magnetic flux density of this static magnetic field similar to the magnetic field generated by motional electric charge varies constantly.

From the above and according to Biot-Savart law, it is known that the source of magnetic field, namely any motional electron and proton generated by all objects, no matter the static objects with motional macroscopic electrons or the motional objects with motional macroscopic electrons, that generate magnetic field only generate static magnetic field at any moment, that is, the motional magnetic field impossibly exists.

\section{Action and Reaction can be in the Same Direction, but Differ in Value}

Figure 2 shows that the isolated negative electric charge $-Q$ (take high voltage discharged electric charge for example) motions at $\mathrm{V}$ along the central line $\mathrm{KJ}$ of current-carrying (current $\mathrm{I}$ ) coil $\mathrm{ABCD}$. According to Biot-Savart law, the magnetic field generated by $-Q$ penetrates through the paper surface above the central line and it is expressed with a small $\odot$ in the figure; if below the central line, it is expressed with a small $\otimes$. According to right-handed screw rule, the magnetic field generated by current-carrying coil ABCD penetrates through the paper inside the coil, it is expressed with a big $\odot$; if it penetrates through the paper outside the paper, it is expressed with a big $\otimes$.

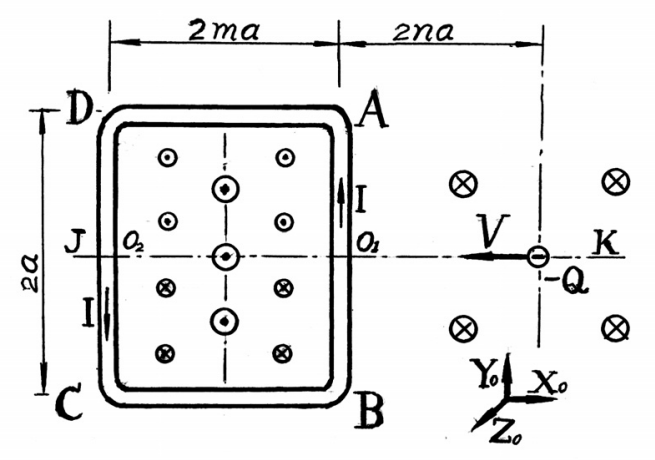

Figure 2. Electric charge motions outside coil

To simplify the formula deduction, in Figure, 2a represents the height of the coil, 2ma represents the width of the coil, 2na represents the distance from $-Q$ to $\mathrm{AB}$ and $X_{0}, Y_{0}$, and $Z_{0}$ represent three unit vectors vertical to each other.

Firstly, we analyze the mutual action between $-Q$ and current-carrying wires DA and BC parallel to V.

By analysis of deduction, we get that the action of magnetic field of current-carrying wire DA to $-Q$ should be the formula as below:

$$
\boldsymbol{F}_{Q}^{D A}=\frac{\mu_{0} I Q V}{2 \pi a}\left(\frac{m+n}{\sqrt{1+4(m+n)^{2}}}-\frac{n}{\sqrt{1+4 n^{2}}}\right)\left(-\boldsymbol{Y}_{0}\right)
$$

Where: $\mu_{0}$ is permeability of vacuum;

$Q$ is carrying capacity of electric charge;

I is current intensity of wires;

The action of magnetic field generated by $-Q$ to current of wire DA is as follows:

$$
\boldsymbol{F}_{D A}^{Q}=\frac{\mu_{0} I Q V}{2 \pi a}\left(\frac{m+n}{\sqrt{1+4(m+n)^{2}}}-\frac{n}{\sqrt{1+4 n^{2}}}\right) \boldsymbol{Y}_{0}
$$

Obviously, $\boldsymbol{F}_{D A}^{Q}=-\boldsymbol{F}_{Q}^{D A}$ 
The action $\boldsymbol{F}_{Q}^{D A}$ of magnetic field generated by current-carrying DA to $-Q$ and action $\boldsymbol{F}_{D A}$ of $-Q$ magnetic field to current of wire DA are same in value but different in direction.

From the similar deduction analysis, we get

$$
\boldsymbol{F}_{B C}^{Q}=-\boldsymbol{F}_{Q}^{B C}
$$

Thus, we come to the conclusion that mutual action between $-Q$ and current-carrying wire parallel to its motional speed $\mathrm{V}$ is same in value but different in direction.

Then, we analyze the mutual action between $-Q$ and current-carrying wires $\mathrm{AB}$ and $\mathrm{CD}$ vertical to $\mathrm{V}$.

It is known from the deduction that the action of magnetic field $\mathrm{AB}$ to $-Q$ is as follows:

$$
\boldsymbol{F}_{Q}^{A B}=\frac{\mu_{0} I Q V}{2 \pi n a \sqrt{1+4 n^{2}}} \boldsymbol{Y}_{0}
$$

The action of magnetic field $\mathrm{CD}$ to $-\mathrm{Q}$ is as follows:

$$
\boldsymbol{F}_{Q}^{C D}=\frac{\mu_{0} I Q V}{2 \pi(m+n) a \sqrt{1+4(m+n)}}{ }^{2}\left(-\boldsymbol{Y}_{0}\right)
$$

From other deduction analysis, we get that the action of magnetic field $-Q$ to $\mathrm{AB}$ is

$$
\boldsymbol{F}_{A B}^{Q}=0
$$

and the action of magnetic field $-Q$ to $\mathrm{CD}$ is

$$
F_{C D}^{Q}=0
$$

The specific deduction process is not to be stated here. We can understand this result from the perspective of physical significance. Firstly, let's see the action of magnetic field $-Q$ to $\mathrm{AB}$ as shown in figure that the upper part $\mathrm{AO}_{1}$ and down part $\mathrm{O}_{1} \mathrm{~B}$ of $\mathrm{AB}$ are same in length and current, but the magnetic field generated by $-Q$ differs in direction. Thus the action of magnetic field $-Q$ to $\mathrm{AO}_{1}$ and the action of magnetic field to $\mathrm{O}_{1} \mathrm{~B}$ are certainly same in value, different in direction and resultant force is zero, namely

$F_{A B}^{Q}=0$, likewise, there is $F_{C D}^{Q}=0$.

As the action $\boldsymbol{F}_{Q}$ of magnetic field of coil to $-Q$ is the summation of actions of magnetic field of current-carrying wires $\mathrm{AB}, \mathrm{BC}, \mathrm{CD}$ and $\mathrm{DA}$ and the action $\boldsymbol{F}_{L}$ of magnetic field $-Q$ to coil is only the summation of the action of magnetic field $-Q$ to wires DA and $\mathrm{CD}\left(\boldsymbol{F}_{A B}^{Q}=0 ; \boldsymbol{F}_{C D}^{Q}=0\right)$, therefore, this is $\left|F_{L}\right| \neq\left|F_{Q}\right|$, namely action and reaction differ in value.

Direction relationship of $F_{L}$ and $F_{Q}$ : firstly look at $F_{L}$, we get to know from the previous:

$\boldsymbol{F}_{L}=\boldsymbol{F}_{D A}^{Q}+\boldsymbol{F}_{C D}^{Q}$, but $\boldsymbol{F}_{D A}^{Q}$ and $\boldsymbol{F}_{C D}^{Q}$ are in $\mathbf{Y}_{\mathrm{o}}$ direction (upward), thus $F_{L}$ is in $\mathbf{Y}_{\mathrm{o}}$ direction; then look at $F_{Q}$ :

$$
\boldsymbol{F}_{Q}=\boldsymbol{F}_{Q}^{D A}+\boldsymbol{F}_{Q}^{B C}+\boldsymbol{F}_{Q}^{A B}+\boldsymbol{F}_{Q}^{C D}
$$

It is already known that $\boldsymbol{F}_{Q}^{A B}$ is in $\mathbf{Y}_{\mathrm{o}}$ direction,

$\boldsymbol{F}_{Q}^{B C}, \boldsymbol{F}_{Q}^{A B}$ and $\boldsymbol{F}_{Q}^{C D}$ are all in $-\mathbf{Y}_{\mathrm{o}}$ direction. It is known from calculation that

$$
\left|\boldsymbol{F}_{Q}^{A B}\right|>\left|F_{\mathrm{Q}}^{\mathrm{BC}}+\mathrm{F}_{\mathrm{Q}}^{\mathrm{AB}}+\mathrm{F}_{\mathrm{Q}}\right|
$$

Namely the directions of $F_{Q}$ and $F_{Q}^{A B}$ are same, namely in $\mathbf{Y}_{\mathrm{o}}$ direction. That is as same as the direction of $F_{L}$.

The detailed calculation will not be stated here. From the perspective of physical significance, we can easily find this result. By referring to Figure 2, the direction of magnetic field at $-Q$ penetrates into the paper and the direction of current generated by $-Q$ is $-\mathrm{V}$ direction. According to Lorentz force law, it is easy to judge that $F_{Q}$ 
is in $\mathrm{Y}_{\mathrm{o}}$ direction (upward).

The above shows that $F_{L}$ and $F_{Q}$ are in $\mathrm{Y}_{\mathrm{o}}$ direction, namely the action of magnetic field $-Q$ to coil and reaction of coil magnetic field to $-Q$ are same in direction. With further analysis and calculation, we get to know that when $\mathrm{n}>10$, no matter the value of aspect ratio $\mathrm{m}$ of coil, there is $\left|\boldsymbol{F}_{O}\right| \approx\left|\boldsymbol{F}_{L}\right|$, namely when the distance from $-Q$ to $\mathrm{AB}$ is 10 times of the length of $\mathrm{AB}$, the mutual action and reaction between $-Q$ and coil are similar in value and direction.

When $-Q$ motions into the coil, there is $\boldsymbol{F}_{A B}^{Q}=0, \boldsymbol{F}_{C D}^{Q}=0$, namely $\boldsymbol{F}_{L}$ and $\boldsymbol{F}_{Q}$ are not same in value, but as the direction of $\boldsymbol{F}_{Q}$ changes from $\mathrm{Y}_{\mathrm{o}}$ to $-\mathrm{Y}_{\mathrm{o}}$, therefore, the direction of $\boldsymbol{F}_{Q}^{A B}$ is certainly in - $\mathrm{Y}_{\mathrm{o}}$ direction, which is contrary to the direction of $\boldsymbol{F}_{L}$ ( $\mathrm{Y}_{\mathrm{o}}$ direction). That is to say when $-Q$ motions into the coil, the mutual action and reaction between $-Q$ and current-carrying coil are not same in value, but differ in direction.

The above analysis shows that under the circumstance of Figure 2, mutual action between $-Q$ and current-carrying coil is not same in value according to principles of electromagnetism. When $-Q$ is in outside of coil, the direction of action and reaction is even same. This analytical result is unreasonable as it is obviously in disagreement with law of action and reaction and goes against energy conservation law.

\section{Magnetic Field Can Have an Infinitely Great Motional Speed}

Current-carrying coil abcd as shown in Figure 3 generates magnetic field outside the coil and annular ring B in the figure represents a magnetic line of this magnetic field. According to principles of electromagnetism, the magnetic field generated by coil abcd can infinitely extend.

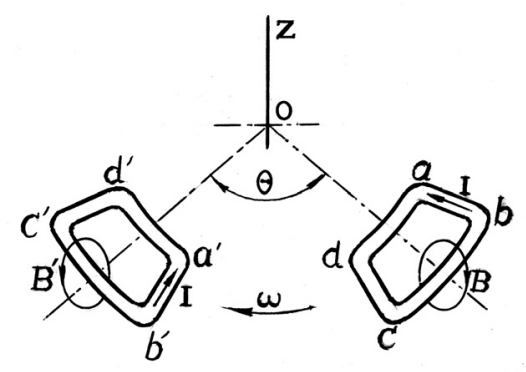

Figure 3. Moving speed of magnetic field can indefinitely great

Now abcd revolves clockwise round axis $\mathrm{OZ}$ at angular speed $\omega$. After $\theta / \omega$ second, abcd revolves by an angle $\theta$ to position $a^{\prime} b^{\prime} c^{\prime} d$ '. As the magnetic field is a substance, magnetic line B certainly revolves by an angle $\theta$ to position $B^{\prime}$ along with abcd (as shown in Figure 3).

Obviously, under this circumstance, the magnetic field of abcd is in the state of revolving with angular speed of $\omega$. In this magnetic field, the motional speed of objects

If $r=c / \omega$, (c-light speed), then $V=\omega c / \omega=c$; if $V>\omega r$, then $V>\mathrm{C}$; if $r \rightarrow \infty$, then $V \rightarrow \infty$. That is to say, the motional speed of magnetic field can not only be greater than light speed, but also can be infinitely great.

Principle of invariance of light speed indicates that light speed is limiting speed in the nature. The deduction that the motional speed of magnetic field previously mentioned can be greater than light speed and even be infinitely great obviously violates the principle of invariance of light speed.

It may be questioned that according to the above deduction method, the motional speed of surrounding magnetic field can also be greater than light speed and even be infinitely great when any an electrified object revolves. This possibility does not exist, because the electric field of electron and proton is field with sources, in which no revolution exists. As shown in Figure 4, electric charge $Q$ revolves at angular speed $\omega$ to $Q^{\prime}$, electric fluxline $\mathrm{E}$ of Q changes to $E^{\prime}$ when it revolves to $Q^{\prime}$. The positions of $E$ and $E^{\prime}$ are different, but the direction of electric field does not change. That is to say, when any an electric charge is revolving, its surrounding electric field only moves but not revolves. When an electrified object composed of many electric charges is revolving, the electric field of each electron and proton on this electrified object only moves without revolution, naturally, the entire electrified object has no revolving electric field and consequently the motional speed of electric field cannot be greater than light speed. 


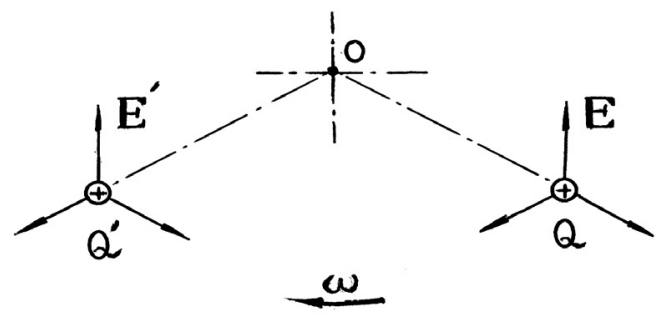

Figure 4. Electric field only moves without revolution

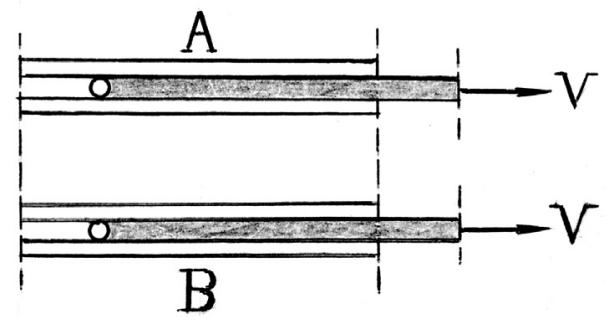

Figure 5. Interaction of parallel current-carrying wire

\section{The Same Physical Phenomenon Can Be Explained Conversely in Different Inertia Systems}

We vividly look on current-carrying wire as a pipeline in which free electrons flow. The fluid in the middle of it represents free electron and pipeline represents the proton with equivalent quantity of electric charge of free electron. In Figure 5, pipelines A and B represent a segment of two equivalent current-carrying wires of infinite length. The average flowing speed of free electron in pipelines A and $\mathrm{B}$ is $\mathrm{V}$.

According to principle of electromagnetism, free electron that motions at speed $\mathrm{V}$ in pipeline $\mathrm{A}$ (or $\mathrm{B}$ ) generate a magnetic field, while free electron that motions at speed $\mathrm{V}$ in pipeline $\mathrm{B}$ (or $\mathrm{A}$ ) traverses this magnetic field and is subject to Ampere force which makes two pipelines attract each other.

However, relevant tube moves at speed $\mathrm{V}$, that is the stationary people relevant to free electron thinks that tube $\mathrm{A}$ (or B) containing positive proton moves at speed - $\mathrm{V}$ and produces magnetic field; tube B (or A) goes through this magnetic field at speed $-\mathrm{V}$ and receives ampere force, which makes two tubes attract each other.

The analytical results of the above two analytical methods are identical, but each thinks that the sources of generating magnetic field are contrary and objects subjected to force are contrary too.

In addition, people who think that free electron motions at speed $\mathrm{V} / 2$ produces magnetic field and receives ampere force, while tube $\mathrm{A}$ and $\mathrm{B}$ positively charged moving at speed $-\mathrm{V} / 2$ produces magnetic field and receives ampere force too.

As to the same physical phenomenon, it is naturally unscientific that different observers have different explanations.

\section{There is no Uniform Scientific Explanation for Deflection of High-Speed Charged Particle in Magnetic} Field

It is a general knowledge of physics that there is deflection when electrified particle goes through magnetic field. Suppose electrified particle goes through magnetic field along direction $\mathrm{X}$ at speed $\mathrm{V}$ after time $\mathrm{t}$, the expression of deflection distance in direction $\mathrm{Y}$ is generally written as follows:

$$
Y=\frac{F}{2 M} t^{2}
$$

Where, $\mathrm{F}=$ acting force that particle received in direction $\mathrm{Y} ; \mathrm{M}=$ static mass of particle

The formula (1) is deduced under the condition that direction F does not change. Speed V and direction of F will 
change when particle goes through magnetic field, but $\mathrm{t}$ is generally minute and changes of $\mathrm{F}$ and $\mathrm{V}$ are very small. The error of $Y$ can be ignored.

The experiment shows that formula (1) is tenable only when $\mathrm{V}$ is smaller; when $\mathrm{V}$ is larger, the actual deflection distance of Y should be as follows:

$$
Y=\frac{F}{2 M} t^{2} \sqrt{1-V^{2} / C^{2}}
$$

Where, $\mathrm{C}=$ light speed

Now the simplest explanation is that according to special relativity, when object motions, its mass should be: $M^{\prime}=M \sqrt{1-V^{2} / C^{2}}$. Substitute the static mass $M$ with $M^{\prime}$ into formula (1), then we get formula (2) conforming to the reality.

There are other two methods that deduce formula (2) by analyzing the variation of momentum of particle.

One method is to suppose that migration velocity of particle in direction $\mathrm{Y}$ is $u$, the mass of particle $M^{\prime}=M \sqrt{1-V^{2} / C^{2}}$. The momentum of particle in direction $\mathrm{Y}: \quad P=M^{\prime} u=M u / \sqrt{1-V^{2} / C^{2}}$.

$$
\begin{gathered}
F=\frac{d p}{d t}=\frac{M}{\sqrt{1-V^{2} / C^{2}}} \frac{d u}{d t} \\
\frac{d u}{d t}=\frac{F \sqrt{1-V^{2} / C^{2}}}{M}
\end{gathered}
$$

Therefrom, we acquire the deflection distance as shown in formula (2).

$$
Y=\int_{0}^{t} d t \int_{0}^{t} \frac{d u}{d t} d t=\frac{F}{2 M} t^{2} \sqrt{1-V^{2} / C^{2}}
$$

It can be found out that the key of this analytical method substitutes $M$ with $M^{\prime}$, which has no substantial difference with formula (1) which directly substitutes $M$ with $M^{\prime}$.

As to another method, there is detailed explanation of it in Berkeley physics (Bersell, 1978) and its fundamental starting point is described as follows. Set $\Delta \tau$ as particle's clock time and set mass as constantly static mass $M$. $\Delta Y$ represents the displacement of particle in direction $Y$ during time $\Delta \tau$. Momentum $P=M \Delta Y / \Delta \tau$. The first and second analytical methods mentioned above use the conversion of mass and the analytical method in Berkeley physics uses the conversion of time. According to special relativity, however, the mass, time and length of particle motioning at high speed convert simultaneously. The acceleration experiment of particle verifies that $\mathrm{M}$ converts Disintegration experiment of meson $\mu$ and $\pi$ verifies that $\mathrm{t}$ varies too. As for the deflection analysis of particle mentioned above, it is necessary to adopt mass conversion $M^{\prime}=M \sqrt{1-V^{2} / C^{2}}$ and time conversion $t^{\prime}=t \sqrt{1-V^{2} / C^{2}}$. Substitute these two conversions $M^{\prime}$ and $t^{\prime}$ into formula (1), we have

$$
Y=\frac{F}{2 M^{\prime}} t^{\prime}=\frac{F}{2 M}\left(1-V^{2} / C^{2}\right)^{3 / 2}
$$

Obviously, formula (3) does not conform to the experimental result. Could it be said that the right analytical method is only to unilaterally convert mass and time? General knowledge tell us that unilateral analytical method is unscientific and it is not proper to unilaterally convert mass and time only in analyzing particle deflection.

Of course, there is another possibility that force $\mathrm{F}$ changes too. It can be found from Formula (3) that the result in Formula (2) can be obtained only by $F^{\prime}=F /\left(1-\mathrm{V}^{2} / \mathrm{C}^{2}\right)$, however, the conversion result of such force does not exist.

In summary, it is hard to find a satisfactory explanation for the deflection result of high-speed electrified particle in magnetic field according to the principles of electromagnetism.

\section{Coexistence of Static Magnetic Field with Static Electric Field Cannot Be Explained}

In 2006, the author laboratory report "Coexistence of static magnetic field with static electric field" (Yao, 2006) was published in the volume 3 of Physics and Engineering. The following is a brief introduction to this report.

As shown in Figure 6, A is a solid NdFeB permanent magnet with side length of $20 \mathrm{~mm}$. the magnetic induction of its surface is about $0.8 \mathrm{~T}$. B is identical with $\mathrm{A}$ in size and its material is also NdFeB, but as it is not magnetized, it is not a permanent magnet. $\mathrm{D}$ is an induced copper disc, which functions to receive electrostatic 
induction of electrified object making P at the bottom end of strip of tin foil induct electric charge with same nature as electrified object. Through adjustment, D can move from left to right and from forward to backward; strip of tin foil can move upward and downward making P be able to locate in an arbitrary position between A and $\mathrm{B}$. L and $\mathrm{R}$ are two large plane metal plates. After being energized, a well-distributed electric field can be produced in the middle area between these two plane metal plates. If the direction of switch is changed, the direction of electric field between $\mathrm{L}$ and $\mathrm{R}$ metal plates will consequently changed.

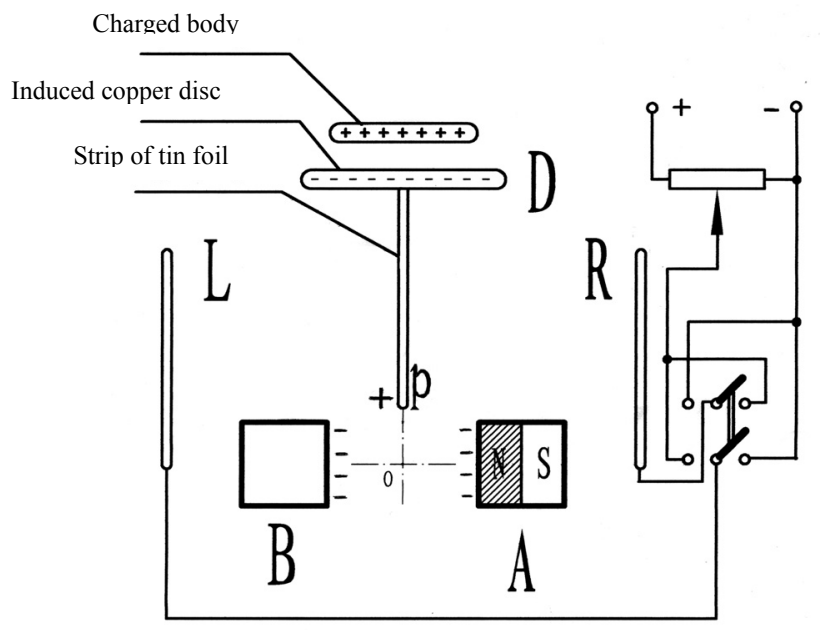

Figure 6. Schematic diagram of test method

See Figure 6, when $\mathrm{P}$ is charged, the surface of $\mathrm{P}$ close to A will have induced charge which will generate electric field. Define this electric field as induced electric field. B functions to generate an induced electric field at $\mathrm{P}$ point, which is identical with the induced electric field of A in value but contrary in direction, making them offset mutually.

Generally, $\mathrm{P}$ is not exactly in the middle place between A and B. if $\mathrm{P}$ is closer to A, then the induced electric field of $\mathrm{A}$ at $\mathrm{P}$ will be stronger than that of $\mathrm{B}$ at $\mathrm{P}$. No matter $\mathrm{P}$ is positively or negatively charged, the direction of its electric field stress will point to A and $\mathrm{P}$ will certainly move toward A; conversely, if $\mathrm{P}$ is closer to $\mathrm{B}$, then $\mathrm{P}$ will certainly move toward $\mathrm{B}$. that is to say, in the event that $\mathrm{P}$ deviates from the middle place, $\mathrm{P}$ will point to an object close to it no matter it is positively or negatively charged so long as A does not generate electric field independently.

In the circumstance that $\mathrm{P}$ is in middle place between $\mathrm{A}$ and $\mathrm{B}$, if $\mathrm{A}$ generates an electric field, a positive electric field for example, at point $\mathrm{P}$ due to some reason, then the direction of electric field will point from $\mathrm{A}$ to $\mathrm{P}$. At the time, the induced electric field of $\mathrm{A}$ and $\mathrm{B}$ offsets each other at point $\mathrm{P}$ and only positive electric field generated by $\mathrm{A}$ acts on point $\mathrm{P}$. If $\mathrm{P}$ is positively charged, the direction of electric field stress that $\mathrm{P}$ receives is from right to left; if $\mathrm{P}$ is negatively charged, the direction of electric field stress that $\mathrm{P}$ receives is from left to right, namely, the direction of movement when $\mathrm{P}$ is positively charged is contrary to the direction of movement when $\mathrm{P}$ is negatively charged. Likewise, if A generates a negative electric field at $\mathrm{P}$, conversely, $\mathrm{P}$ is positively charged and the direction of electric field stress it receives moves from left to right; $\mathrm{P}$ is positively charged and the direction of electric field stress it receives moves from

It is known from the above discussion that in the circumstance that $\mathrm{P}$ is in the middle position between $\mathrm{A}$ and $\mathrm{B}$, if A generates an electric field at $\mathrm{P}$, there is the conclusion that the direction of movement when $\mathrm{P}$ is positively charged is contrary to the direction of movement when $\mathrm{P}$ is negatively charged. That is to say, so long as we find that the direction of movement when $\mathrm{P}$ is positively charged is contrary to the direction of movement when $\mathrm{P}$ is negatively charged, it can be determined that A generates an electric field by itself, at the same time, the direction of this electric field can be determined.

Of course, under normal circumstance, it is hard to exactly adjust $\mathrm{P}$ to the middle position between $\mathrm{A}$ and $\mathrm{B}$, but so long as the distance that $\mathrm{P}$ deviates from the middle position is not long and the electric field generated by $\mathrm{A}$ at $\mathrm{P}$ is very strong, then the field strength of the self-induced electric field of $\mathrm{A}$ at $\mathrm{P}$ can be markedly larger than the total electric field strength of induced electric fields A and B, thus there is still the result that the direction of 
movement when $\mathrm{P}$ is positively charged is contrary to the direction of movement when $\mathrm{P}$ is negatively charged.

The measurement result shows that the central zone of two surfaces of permanent magnet $\mathrm{N}$ and $\mathrm{S}$ and central zone of four sides between permanent magnet $\mathrm{N}$ and $\mathrm{S}$ have no self-induced electric field or it is very minute; but there is static negative electric field near the four edges of permanent magnet $\mathrm{N}$ and $\mathrm{S}$ and there is static positive electric field near four edges of four sides.

Through the adjustment of applied voltage and positive and negative between $\mathrm{L}$ and $\mathrm{R}$, an electric field that is equivalent to self-induced electric field of magnet in value but different in direction can be generated at $\mathrm{P}$, making $\mathrm{P}$ positively or negatively charged no longer move toward the contrary direction, at this time the applied field strength at $\mathrm{P} \mathrm{b}$ and positive and negative between $\mathrm{L}$ and $\mathrm{R}$, an electric field that is equivalent to self-induced electric field of magnet in value but different in direction can be generated at $\mathrm{P}$, making $\mathrm{P}$ positively or negatively charged no longer move toward the contrary direction, at this time the applied field strength at $\mathrm{P}$ between $\mathrm{L}$ and $\mathrm{R}$ equals the self-induced electric field strength of permanent magnet at $\mathrm{P}$. It is roughly determined that when $\mathrm{P}$ is $5 \mathrm{~mm}$ away from the edge of $\mathrm{N}$ or $\mathrm{S}$, its field strength is $-240 \mathrm{~V} / \mathrm{m}$; when $\mathrm{P}$ is $10 \mathrm{~mm}$ away from the edge of $\mathrm{N}$ or $\mathrm{S}$, its field strength is $-130 \mathrm{~V} / \mathrm{m}$; when $\mathrm{P}$ is $5 \mathrm{~mm}$ away from the edge of side, its field strength is $+190 \mathrm{~V} / \mathrm{m}$; when when $\mathrm{P}$ is $10 \mathrm{~mm}$ away from the edge of side, its field strength is $+95 \mathrm{~V} / \mathrm{m}$.

According to Coulomb's law of static electric field, there is static electric field only around electrified object. Permanent magnet is not an electrified object and the existence of its static electric field is a phenomenon is hard to be explained by electromagnetism.

\section{References}

Lifshitz, E. M. (1978). Theory of Field. China: People's Education Press.

Hou, S. Y. (1984). General Physics. China: People's Posts and Telecommunications Press.

Physics Compilation Group of Fudan University. (1985). "Physics" High Education Press.

Wu, S. H. (1987). Fundamentals on Theory of Relativity. Press of Science and Technology of Shaanxi Province.

Yao, K. X. (2006). Physics and Engineering. Tsinghua University Press. Mar., 44.

Bersell, E. M. (1978). Berkeley Physics Tutorial. China: China Science Press. 